\title{
Non-coding RNA 886 promotes renal cell carcinoma growth and metastasis through the Janus kinase 2/signal transducer and activator of transcription 3 signaling pathway
}

\author{
JUN LEI $^{1,2^{*}}$, JU-HUA XIAO ${ }^{1,3^{*}}$, SHOU-HUA ZHANG ${ }^{2}$, ZHI-QIANG LIU ${ }^{2}$, \\ KAI HUANG ${ }^{4}$, ZHI-PENG LUO ${ }^{4}$, XIN-LAN XIAO ${ }^{5}$ and ZHENG-DONG HONG ${ }^{1}$
}

${ }^{1}$ Department of Urology, The Second Affiliated Hospital of Nanchang University; ${ }^{2}$ Department of General Surgery, Jiangxi Provincial Children's Hospital; ${ }^{3}$ Department of Ultrasound, Jiangxi Provincial Maternal and Child Health Hospital, Nanchang, Jiangxi 330006; ${ }^{4}$ Department of Gastrointestinal Surgery, Jiangxi Provincial Cancer Hospital, Nanchang, Jiangxi 330029;

${ }^{5}$ Department of MRI, The Second Affiliated Hospital of Nanchang University, Nanchang, Jiangxi 330006, P.R. China

Received August 17, 2016; Accepted May 12, 2017

DOI: $10.3892 / \mathrm{mmr} .2017 .7093$

\begin{abstract}
Non-coding RNA 886 (nc886) has been suggested to serve tumor-suppressing roles in several cancer cells. However, the expression pattern of nc886 and its function in renal cell carcinoma (RCC) has not been reported until now. The present study aimed to examine the expression of nc886 in human RCC tissues and to investigate the role of nc886 in RCC cell proliferation, apoptosis and invasion in vitro. Furthermore, whether nc886 exerts its function on RCC via Janus kinase 2/signal transducer and activator of transcription 3 (JAK2/STAT3) signaling was investigated. It was demonstrated that nc886 is overexpressed in human RCC tissues compared with normal tissues, as determined by reverse transcription-quantitative polymerase chain reaction analysis. The nc 886 mimic and inhibitor were transfected into the A-498 cells to overexpress or knock down nc886 expression. Cell proliferation, cell apoptosis rate and cell invasion ability were determined by MTT, flow cytometry and Transwell-Matrigel invasion assays. The results demonstrated that nc886 overexpression promotes A-498 cell proliferation and invasion, and inhibits cell apoptosis, while nc886 knockdown resulted in the opposite effects. Furthermore, nc886 could activate the JAK2/STAT3 signaling pathway in A-498 cells. AG490, an inhibitor of JAK2,
\end{abstract}

Correspondence to: Dr Zheng-Dong Hong, Department of Urology, The Second Affiliated Hospital of Nanchang University, 1 Minde Road, Nanchang, Jiangxi 330006, P.R. China

E-mail: hongzhengdong57@sina.com

Dr Xin-Lan Xiao, Department of MRI, The Second Affiliated Hospital of Nanchang University, 1 Minde Road, Nanchang, Jiangxi 330006, P.R. China

E-mail: xiao_x12000@126.com

*Contributed equally

Key words: non-coding RNA 886, renal cell carcinoma, JAK2/STAT3, growth, metastasis could attenuate the effects of nc886 on cell proliferation, apoptosis and invasion. In conclusion, to the best of our knowledge, the present study for the first time revealed the expression profile and the tumor-promoting role of nc886 in RCC. nc886 affects RCC cell proliferation, apoptosis and invasion at least partially via the activation of JAK2/STAT3 signaling. This study may provide a useful therapeutic target for RCC.

\section{Introduction}

Renal cell carcinoma (RCC) accounts for 2-3\% of adult malignancies, and is the second most common malignancy in the urinary system $(1,2)$. Surgical resection is the best treatment for RCC, and the 5-year survival rate is $65-90 \%$ (3); however, the 5-year survival rate is lowered considerably following the development of metastatic cancer (4-6). Therefore, elucidating the mechanisms involved in RCC metastasis is essential to help in developing novel therapeutic targets.

Non-coding (nc)RNAs are RNA transcripts that are not translated into proteins. Some ncRNAs serve diverse functions in many cellular processes, and the regulatory ncRNAs have attracted much attention in the past decades (7). ncRNA886 (nc886, also termed pre-miR-886 or CBL3), a newly discovered ncRNA (8), was originally known as a precursor microRNA (9) or a vault RNA (vtRNA) in the vault complex $(10,11)$. However, numerous researches have demonstrated that nc886 is barely processed into mature microRNAs, and is not in the vault complex; therefore, it has been renamed as nc886 (8). The expression of nc 886 is silenced in many malignancies by $\mathrm{CpG}$ DNA hypermethylation (12-15). nc886 controls protein kinase $\mathrm{R}$ activity and has been suggested to serve tumor-suppressing roles in several cancer cells, including cholangiocarcinoma, esophageal squamous cell carcinoma (ESCC) and gastric cancer $(8,9,16)$. However, Kong et al (17) demonstrated that nc886 vtRNA2-1-5p is overexpressed in human cervical squamous cell carcinomas, and vtRNA2-1-5p has oncogenic activity associated with the progression of cervical cancer. Until now, the expression pattern of nc886 and its function in renal cell carcinoma has not been reported. 
The Janus kinase 2/signal transducer and activator of transcription 3 (JAK2/STAT3) signaling pathway has received much attention in recent years. JAK2/STAT3 signaling activation occurs frequently in cancer cells, and the persistent constitutive activation of JAK2/STAT3 is associated with cell proliferation, migration and angiogenesis, thus contributing to cancer development (18-21).

The present study examined the expression of nc886 in human RCC tissues and investigated the role of nc886 in RCC cell proliferation, apoptosis and invasion in vitro. Furthermore, whether nc886 exerts its function on RCC via JAK2/STAT3 signaling was examined. This study may provide the first evidence on the roles and mechanism of nc886 in RCC growth and metastasis.

\section{Materials and methods}

Tissue samples. The present study was approved by the Ethics Committee of The Second Affiliated Hospital of Nanchang University (Nanchang, China). Informed consent was obtained from all patients prior to sample collection. A total of 20 patients with RCC were included in this study (male, 12; female, 8; age, 35-72). At the time of diagnosis, 11 patients had stage I and stage II, whereas the other 9 patients had stage III and IV RCC. The tumor tissues and the adjacent normal tissues were obtained from surgery and frozen in liquid nitrogen.

Cell culture and transfection. A-498 cells were purchased from the American Type Culture Collection (Manassas, VA, USA), and cultured in Dulbecco's modified Eagle's medium (DMEM; Invitrogen; Thermo Fisher Scientific, Inc., Waltham, MA, USA) supplemented with $10 \%$ (v/v) fetal bovine serum (FBS; Gibco; Thermo Fisher Scientific, Inc.). Cells were maintained at $37^{\circ} \mathrm{C}$ in a humidified atmosphere of $5 \% \mathrm{CO}_{2}$. AG490 was obtained from Sigma-Aldrich; Merck KGaA (Darmstadt, Germany) and dissolved in dimethyl sulfoxide (DMSO; Amresco, LLC, Solon, OH, USA). AG490 $(10 \mu \mathrm{M})$ was used to treat cells for $24 \mathrm{~h}$, and the experiement was repeated three times. The nc886 mimic and inhibitor were purchased from Shanghai GenePharma Co., Ltd. (Shanghai, China). The cells were transfected with the nc886 mimic or nc886 inhibitor at a final concentration of $50 \mathrm{nM}$ using Lipofectamine 2000 reagent (Invitrogen; Thermo Fisher Scientific, Inc.).

Reverse transcription-quantitative polymerase chain reaction (RT-qPCR). Total RNA was extracted from the tissues and cells using TRIzol reagent (Invitrogen; Thermo Fisher Scientific, Inc.). Total RNA $(1 \mu \mathrm{g})$ was reverse-transcribed into cDNA using the Transcriptor First Strand cDNA Synthesis kit (Roche Diagnostics, Basel, Switzerland). The following primers were used: nc886, 5'-CGGGTCGGAGTTAGCTCA-3' (forward) and 5'-TGCGAATACCTCGGACCCTG-3' (reverse); U6 snRNA, 5'-ATTGGA ACGATACAGAGA AGATT-3' (forward) and 5'-GGAACGCTTCACGAATTTG-3' (reverse). The qPCR assay was performed on an ABI 7000 real-time PCR system (Applied Biosystems; Thermo Fisher Scientific, Inc.) using the SYBR Green PCR kit (Applied Biosystems; Thermo Fisher Scientific, Inc.). The reaction was conducted using the following conditions: $95^{\circ} \mathrm{C}$ for $10 \mathrm{~min}$, followed by
40 cycles of $95^{\circ} \mathrm{C}$ for $15 \mathrm{sec}, 60^{\circ} \mathrm{C}$ for $1 \mathrm{~min}$, and $72^{\circ} \mathrm{C}$ for $30 \mathrm{sec}$. The expression of nc886 was normalized to a control gene (U6), and relative induction was calculated using the $2^{-\Delta \Delta \mathrm{Cq}}$ method (22).

Western blotting. Cells were lysed by radioimmunoprecipitation assay buffer (Sangon Biotech, Co., Ltd., Shanghai, China), and the protein concentrations were measured using a Bicinchoninic Acid Protein Assay kit (Beyotime Institute of Biotechnology, Shanghai, China). Total proteins $(20 \mu \mathrm{g})$ were resolved by $12 \%$ SDS-PAGE at $200 \mathrm{~V}$ for $90 \mathrm{~min}$, and transferred to polyvinylidene fluoride membranes (EMD Millipore, Billerica, MA, USA). After blocking in 5\% non-fat milk at $4^{\circ} \mathrm{C}$ overnight, the membranes were incubated with the following specific monoclonal antibodies at $4^{\circ} \mathrm{C}$ overnight: Mouse anti-phosphorylated (p)-Stat3 (Tyr705; 1:500; cat. no. 4113), mouse Anti-Stat3 (1:400; cat. no. 9139), rabbit anti-p-JAK2 (Tyr1007; 1:400; cat. no. 4406) and rabbit anti-JAK2 (1:800; cat. no. 3230). These antibodies were all purchased from Cell Signaling Technology, Inc. (Cambridge, MA, USA). After washing with Tris-buffered saline containing $0.05 \%$ Tween-20, the membranes were incubated with horseradish peroxidase (HRP)-conjugated Affinipure goat anti-mouse IgG (H+L; 1:2,000; cat. no. SA00001-1; Wuhan Sanying Biotechnology, Wuhan, China) and HRP-conjugated Affinipure goat anti-rabbit IgG $(\mathrm{H}+\mathrm{L}$; 1:5,000; cat. no. SA00001-2; Wuhan Sanying Biotechnology) secondary antibodies at $37^{\circ} \mathrm{C}$ for $1 \mathrm{~h}$. The immunoreactive proteins were detected using an enhanced chemiluminescence western blotting kit (Thermo Fisher Scientific, Inc.), and the band intensity was quantified using Image J v1.52 software (imagej.nih.gov/ij/; National Institutes of Health, Bethesda, MD, USA).

MTT assay. An MTT assay was performed to determine cell proliferation. The cells were seeded into 96 -well plates at the density of 1,500 cells/well. After culturing for the indicated times, MTT solution $(0.5 \mathrm{mg} / \mathrm{ml}$; Beyotime Institute of Biotechnology) was added to each well and incubated at $37^{\circ} \mathrm{C}$ for $4 \mathrm{~h}$. The formazan crystals were dissolved by DMSO. The cell viability was detected by measurement of the absorbance at $570 \mathrm{~nm}$ using a microplate reader (Multiskan FC; Thermo Fisher Scientific, Inc., Waltham, MA, USA).

Flow cytometry (FCM) assay. FCM assay was performed to determine cell apoptosis. The cells were harvested and washed with PBS. Subsequently, the cells were dual-stained with Annexin V-fluorescein isothiocyanate (FITC) and propidium iodide (PI) using an Annexin V-FITC and PI apoptosis kit (Nanjing KeyGen Biotech Co., Ltd., Nanjing, China) according to the manufacturer's protocol. After incubation at room temperature for $5 \mathrm{~min}$ in the dark, the cells were immediately analyzed by flow cytometry (CytoFLEX; Beckman Coulter Inc., Brea, CA, USA) using CytExpert 1.2 analysis software (Beckman Coulter Inc.).

Transwell-Matrigel invasion assay. A Transwell-Matrigel invasion assay was performed to determine cell invasion ability. The Transwell chambers (Corning, Tewksbury, MA, USA) with $8-\mu \mathrm{m}$ pore polyethylene membranes were 
pre-coated with Matrigel (BD Biosciences, Franklin Lakes, NJ, USA). The A-498 cells were suspended in serum-free medium, and placed in the upper chamber of inserts at the density of $5 \times 10^{4}$ cells $/ \mathrm{ml}$. The lower chamber contained culture medium with $10 \% \mathrm{FBS}$. The cells were incubated at $37^{\circ} \mathrm{C}$ for $12 \mathrm{~h}$, and they were then fixed and stained with hematoxylin (Beyotime Institute of Biotechnology). The invaded cells were observed and counted using an inverted microscope (37XC; Shanghai Optical Instrument Co., Ltd., Shanghai, China).

Statistical analyses. Data are expressed as the mean \pm standard deviation of at least three independent experiments. Statistical analyses were performed on SPSS 19.0 software (IBM SPSS, Armonk, NY, USA). The statistical significance was assessed by Student's t-test or one-way analysis of variance followed by the least significant difference test. $\mathrm{P}<0.05$ was considered to indicate a statistically significant difference.

\section{Results}

Expression of nc886 in human RCC tissues. The expression of nc886 in human RCC tissues and the adjacent normal tissues was assessed using RT-qPCR analysis. It was revealed that the mRNA expression level of nc886 was significantly increased in the RCC tissues compared with the adjacent normal tissues, particularly in stage III-IV cancer, compared with RCC at stage I-II (Fig. 1).

Effect of nc886 on RCC cell proliferation. The nc886 mimic and the nc886 inhibitor were transfected into the A-498 RCC cell line, and the results of RT-qPCR analysis indicated that nc886 mRNA expression levels were significantly downregulated in A-498 cells following transfection with the nc886 inhibitor. A 3-4-fold increase of nc886 mRNA was detected in nc886 mimic-transfected A-498 cells (Fig. 2).

Subsequently, effect of nc886 on RCC cell proliferation was investigated using MTT assay. As presented in Fig. 3, the nc886 mimic-transfected cells displayed a significantly higher growth rate compared with the control; nc886 knockdown significantly suppressed cell growth.

Effect of nc886 on RCC cell apoptosis. The results from RT-qPCR analysis revealed that there was no significant difference in the cell apoptosis rate between the blank group and the control group. Compared with the control, the cell apoptosis rate was significantly decreased in A-498 cells transfected with the nc886 mimic. Conversely, the cell apoptosis rate was significantly increased in A-498 cells transfected with the nc886 inhibitor (Fig. 4).

Effect of nc886 on RCC cell invasion. A Transwell-Matrigel invasion assay was performed to examine the effect of nc886 on RCC cell invasion. As presented in Fig. 5, the nc886 mimic-transfected cells demonstrated an enhanced cell invasion ability compared with the control cells. By contrast, the nc886 inhibitor significantly reduced the invasive ability of A-498 cells (Fig. 5).

Effect of nc886 on JAK2/STAT3 signaling in RCC cells. Whether nc886 affects JAK2/STAT3 signaling in RCC cells

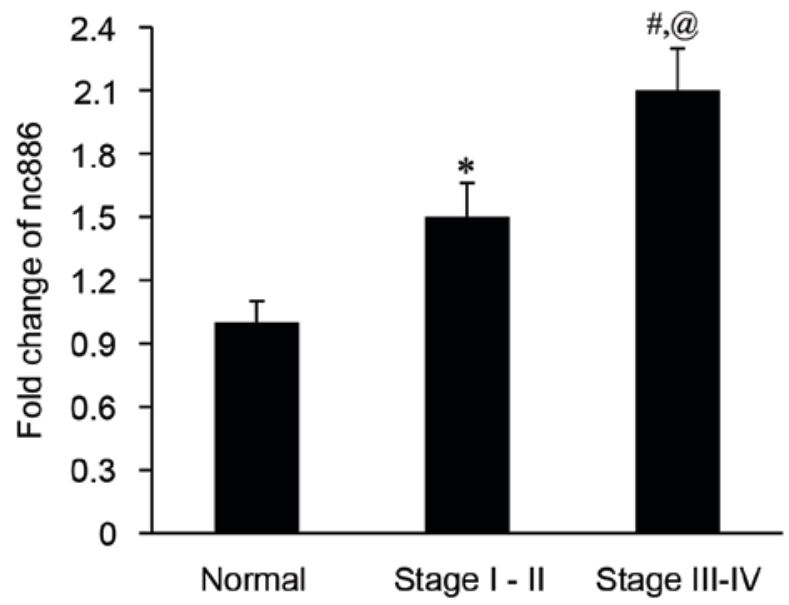

Figure 1. Expression of nc886 in human renal cell carcinoma tissues. Data are expressed as the mean \pm standard deviation. ${ }^{*} \mathrm{P}<0.05$ and ${ }^{\#} \mathrm{P}<0.01$ vs normal; ${ }^{\circledR} \mathrm{P}<0.05$ vs. stage I-II. nc886, non-coding RNA 886.

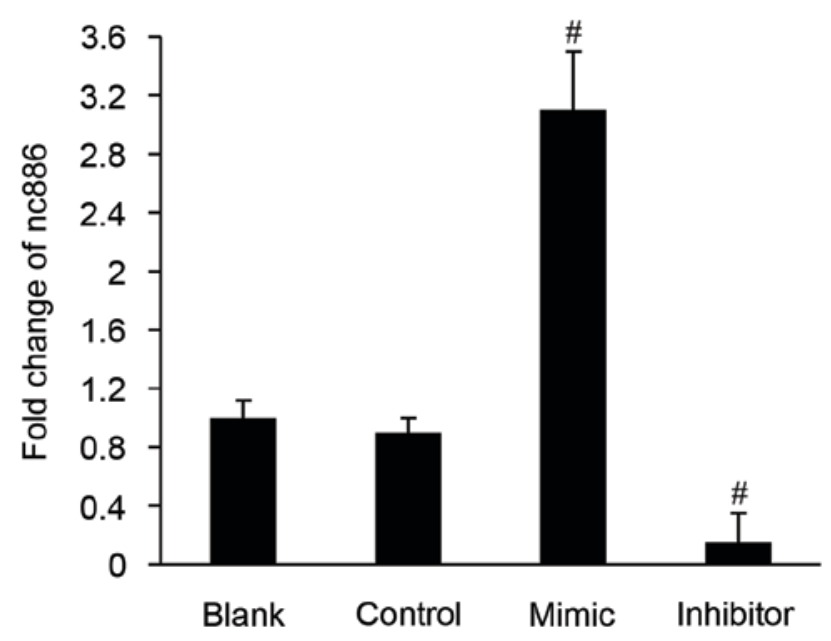

Figure 2. Expression of nc886 in A-498 cells following transfection with the nc886 mimic and the nc886 inhibitor. Data are expressed as the mean \pm standard deviation. ${ }^{~} \mathrm{P}<0.01$ vs. control. nc 886 , non-coding RNA 886.

was subsequently investigated. The results of western blot analysis demonstrated that the nc886 mimic induced phosphorylation of JAK2 at Tyr1007 in A-498 cells. Meanwhile, the phosphorylation level of STAT3 at Tyr705 was elevated in A-498 cells transfected with the nc886 mimic. Furthermore, the phosphorylation levels of JAK2 and STAT3 were significantly decreased in A-498 cells transfected with the nc886 inhibitor (Fig. 6).

JAK2/STAT3 signaling is involved in mediating the effect of nc886 on RCC cell proliferation, apoptosis and invasion. Subsequently, effect of nc886 on RCC cell proliferation, apoptosis and invasion was investigated following inhibition of JAK2/STAT3 signaling. As demonstrated in Fig. 7A, the increased phosphorylation levels of JAK2 and STAT3 by nc886 mimic treatment was abolished by the JAK2 inhibitor AG490. Furthermore, the enhanced cell proliferation and invasion abilities caused by nc 886 mimic was attenuated by AG490. The decreased cell apoptosis rate by nc886 mimic treatment was also reversed by AG490 (Fig. 7B-D). 


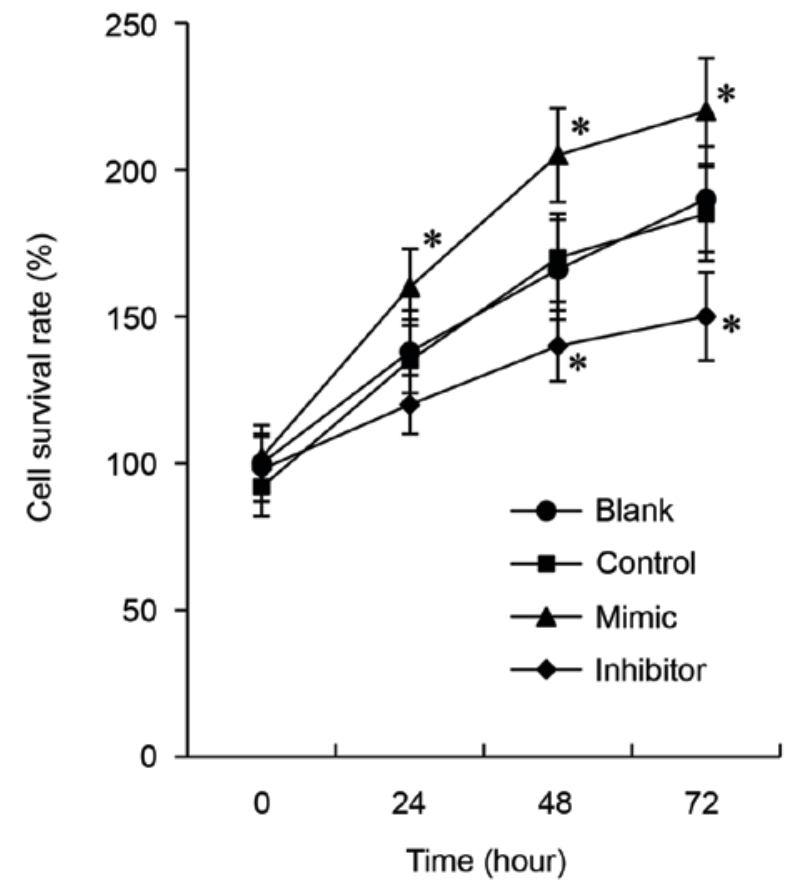

Figure 3. Effect of non-coding RNA 886 on renal cell carcinoma cell proliferation. Data are expressed as the mean \pm standard deviation. ${ }^{*} \mathrm{P}<0.05$ vs. matched time-point control.

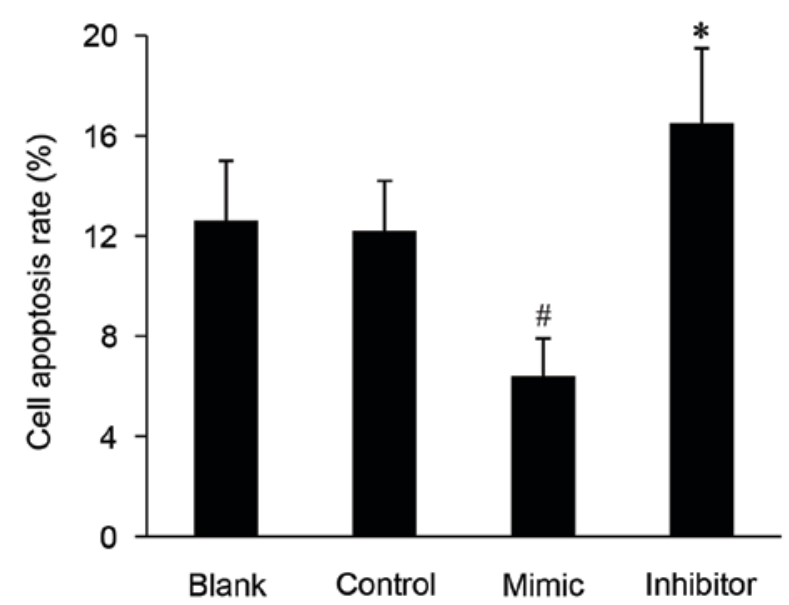

Figure 4. Effect of non-coding RNA 886 on renal cell carcinoma cell apoptosis. Data are expressed as the mean \pm standard deviation. ${ }^{*} \mathrm{P}<0.05$ and ${ }^{\text {"P }}<0.01$ vs. control.

\section{Discussion}

nc886 is encoded on human chromosome $5 \mathrm{q} 31$ and is composed of $\sim 102$ nucleotides. Numerous studies have demonstrated that nc886 is ubiquitously expressed in non-malignant tissues, but is suppressed in many cancer cells of various tissue origins (12-16). The present study observed that nc886 is overexpressed in human RCC tissues compared with normal tissues, and its expression level is positively associated with the stage of RCC. This finding suggested that nc 886 has a specific biological role in RCC.

Previous studies have demonstrated that the low expression of nc886 is significantly associated with poor survival of patients $(12,13)$. In the study by Lee et al, ectopically

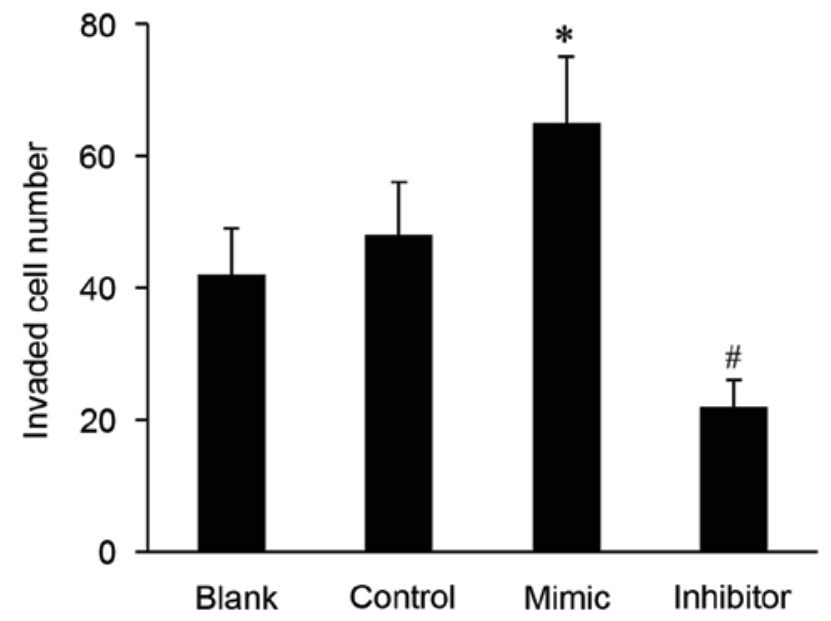

Figure 5. Effect of non-coding RNA 886 on renal cell carcinoma cell invasion. Data are expressed as the mean \pm standard deviation. ${ }^{*} \mathrm{P}<0.05$ and ${ }^{\#} \mathrm{P}<0.01$ vs. control. RCC, renal cell carcinoma.
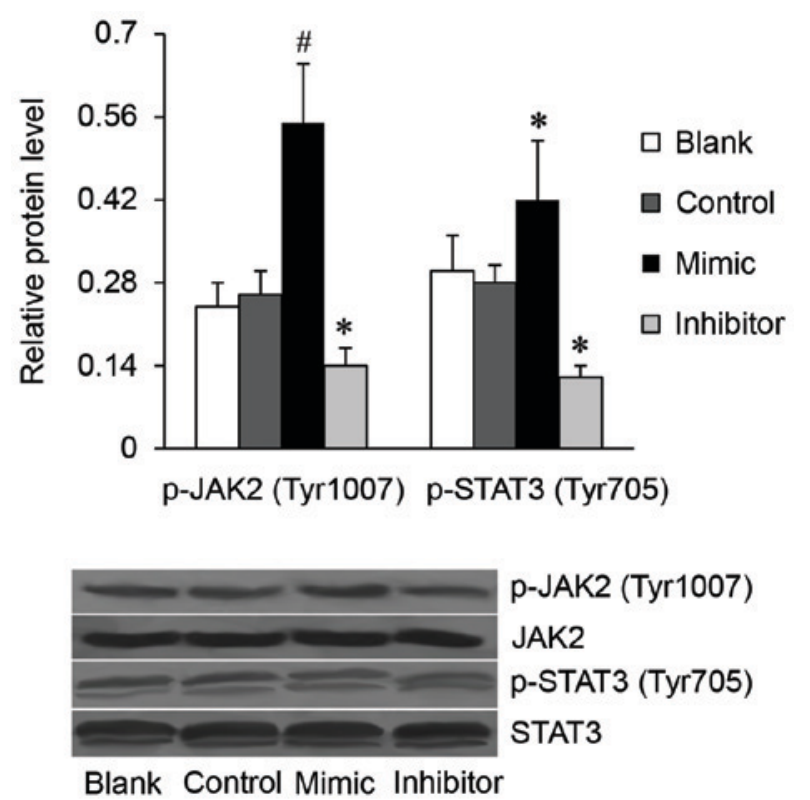

Figure 6. Effect of non-coding RNA 886 on JAK2/STAT3 signaling in renal cell carcinoma cells. Data are expressed as the mean \pm standard deviation. ${ }^{*} \mathrm{P}<0.05$ and ${ }^{\#} \mathrm{P}<0.01$ vs. control of the same protein. JAK2/STAT3, Janus kinase 2/signal transducer and activator of transcription 3; p, phosphorylated; Tyr, tyrosine.

expressed nc886 inhibits gastric cancer cell proliferation (13). Knockdown of nc886 correlates with the induction of oncogenes such as c-Fos, nuclear factor (NF)-кB, and c-Myc (9). nc886 is also suggested to be a potential diagnostic marker in ESCC. It has been demonstrated that nc 886 is epigenetically silenced in human ESCC tissues, and regulates the expression of oncogenes in ESCC (8). In the present study, gain- and loss-of function experiments were performed to determine the role of nc886 in A-498 cell proliferation, apoptosis and invasion. nc 886 overexpression was identified to promote A-498 cell proliferation and invasion, and inhibit cell apoptosis. nc886 knockdown demonstrated the opposite effects on A-498 cell proliferation, apoptosis and invasion. These findings confirmed that nc886 functions as an oncogene in RCC, 
A
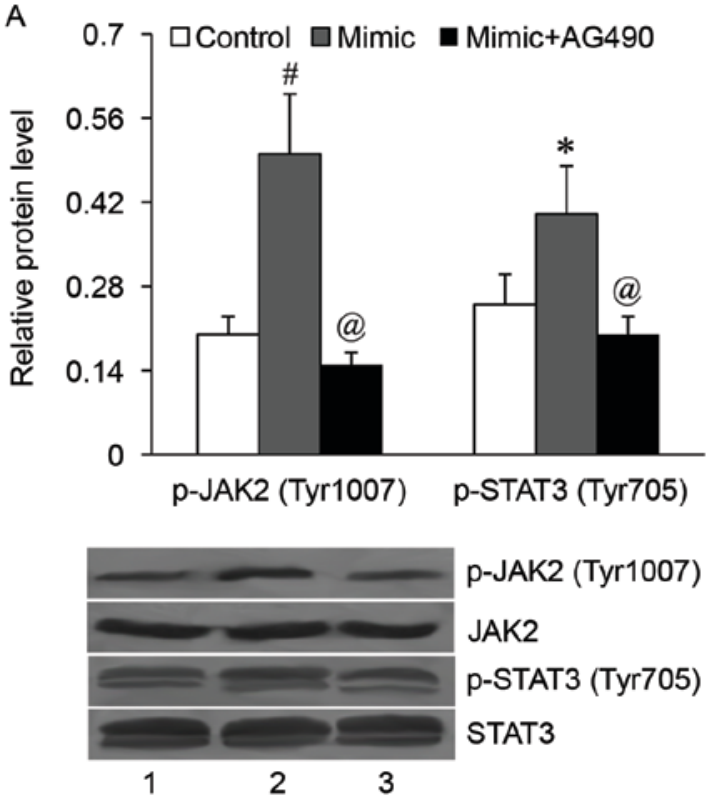

C

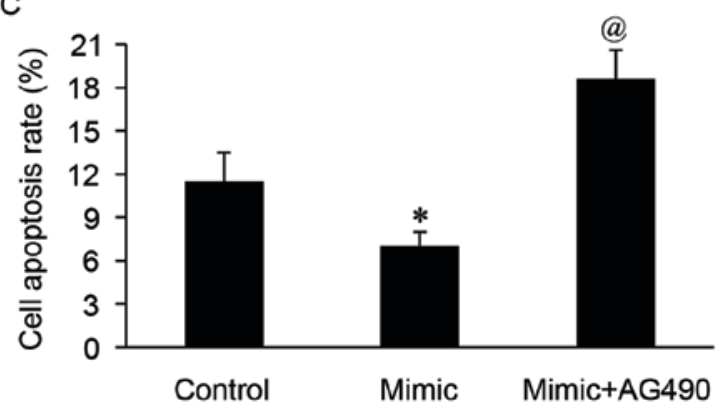

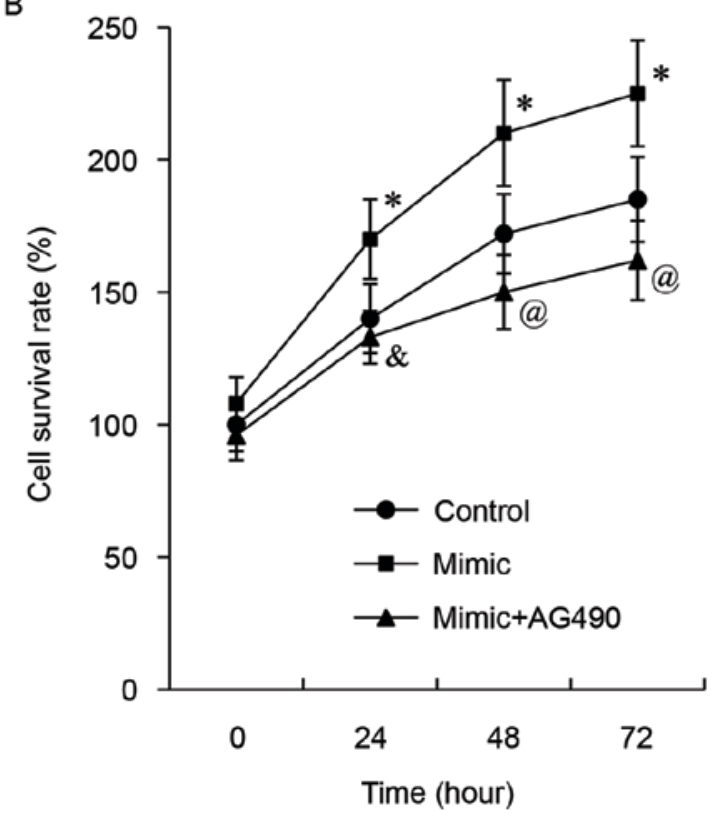

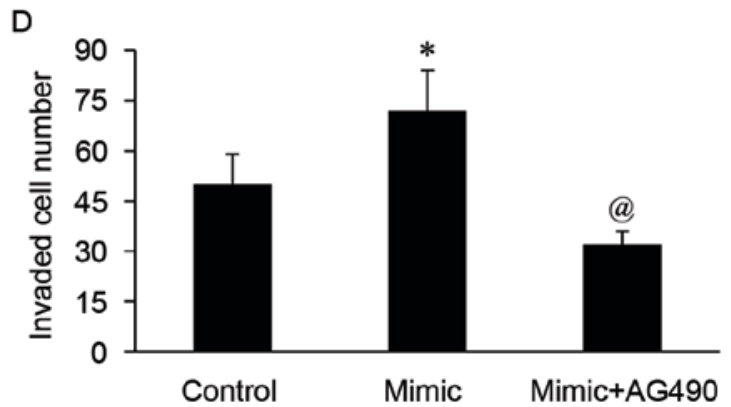

Figure 7. JAK2/STAT3 signaling is involved in mediating the effect of non-coding RNA 886 on renal cell carcinoma cell proliferation, apoptosis and invasion. (A) The relative protein expression levels of p-JAK2 (Tyr1007) and p-STAT3 (Tyr705). Lane 1, Control; lane 2, Mimic; lane 3, Mimic+AG490. (B) Cell proliferation, (C) cell apoptosis rate and (D) cell invasion ability of A-498 cells following transfection with the nc886 mimic and AG490 treatment. Data are expressed as the mean \pm standard deviation. ${ }^{*} \mathrm{P}<0.05$ and ${ }^{\#} \mathrm{P}<0.01$ vs. control; ${ }^{\&} \mathrm{P}<0.05$ and ${ }^{\circledR} \mathrm{P}<0.01$ vs. mimic. JAK2/STAT3, Janus kinase $2 /$ signal transducer and activator of transcription 3; p, phosphorylated; Tyr, tyrosine.

and it was indicated that the function of nc886 appears to be cell-type specific.

The JAK2/STAT3 signaling serves important roles in oncogenesis and tumor growth. JAK 2 binds to the membrane receptors, and STAT3 is recruited to receptor-JAK2 complexes and is phosphorylated by JAK2. The phosphorylated STAT3 translocates into the cell nucleus, and acts as a transcription factor, serving key roles in many cellular processes such as cell growth, apoptosis, angiogenesis and immune escape (23-26). Previous studies have reported that nc886 depletion could activate $\mathrm{NF}-\kappa \mathrm{B}$ in cholangiocarcinoma, ESCC and gastric cancer $(12,13,16)$. In addition, p53 expression is regulated by nc886 in cervical cancer (17). STAT3 is the upstream regulator of $\mathrm{NF}-\kappa \mathrm{B}$ and p53; STAT3 and $\mathrm{NF}-\kappa \mathrm{B}$ functionally interact with each other at many different levels $(27,28)$. The present study further investigated whether nc886 affects RCC cell proliferation, apoptosis and invasion via the JAK2/STAT3 signaling. The results demonstrated that nc886 could activate the JAK2/STAT3 signaling pathway in A-498 cells, indicating that the effect of nc886 on JAK2/STAT3 signaling activation in RCC is different from its effect in cholangiocarcinoma, ESCC and gastric cancer. This inconsistency suggested that the effect of nc886 on JAK2/STAT3 pathway may be cell-type specific. It was hypothesized that nc886 may serve different roles in STAT3 and NF- $\kappa$ B activation in different cancer cells. The direct effect of nc886 on NF- $\mathrm{NB}$ activation in $\mathrm{RCC}$, and the molecular mechanism behind such difference, requires further investigation. Furthermore, in the present study, AG490, an inhibitor of JAK2, attenuated the effects of nc886 on cell proliferation, apoptosis and invasion. Overall, these results demonstrated that JAK2/STAT3 signaling mediates the effects of nc886 on RCC cell proliferation, apoptosis and invasion.

In conclusion, to the best of our knowledge, the present study was the first to reveal the expression profile and tumor-promoting role of nc886 in RCC. Furthermore, the molecular mechanism that nc 886 affects RCC cell proliferation, apoptosis and invasion at least partially via the activation of JAK2/STAT3 signaling was demonstrated. The present study may provide a useful therapeutic target for RCC. 


\section{References}

1. Motzer RJ, Agarwal N, Beard C, Bhayani S, Bolger GB, Carducci MA, Chang SS, Choueiri TK, Hancock SL, Hudes GR, et al: Kidney cancer. J Natl Compr Canc Netw 9: 960-977, 2011

2. Siegel RL, Miller KD and Jemal A: Cancer statistics, 2015. CA Cancer J Clin 65: 5-29, 2015

3. Schöffski P, Dumez H, Clement P, Hoeben A, Prenen H, Wolter P, Joniau S, Roskams T and Van Poppel H: Emerging role of tyrosine kinase inhibitors in the treatment of advanced renal cell cancer: A review. Ann Oncol 17: 1185-1196, 2006

4. Motzer RJ, Bander NH and Nanus DM: Renal-cell carcinoma. N Engl J Med 335: 865-875, 1996.

5. Lam JS, Leppert JT, Belldegrun AS and Figlin RA: Novel approaches in the therapy of metastatic renal cell carcinoma. World J Urol 23: 202-212, 2005.

6. Amato RJ: Renal cell carcinoma: Review of novel single-agent therapeutics and combination regimens. Ann Oncol 16: 7-15, 2005

7. Mattick JS and Makunin IV: Non-coding RNA. Hum Mol Genet 15 Spec No 1: R17-R29, 2006.

8. Lee K, Kunkeaw N, Jeon SH, Lee I, Johnson BH, Kang GY, Bang JY, Park HS, Leelayuwat C and Lee YS: Precursor miR-886, a novel noncoding RNA repressed in cancer, associates with PKR and modulates its activity. RNA 17: 1076-1089, 2011.

9. Landgraf P, Rusu M, Sheridan R, Sewer A, Iovino N, Aravin A, Pfeffer S, Rice A, Kamphorst AO, Landthaler M, et al: A mammalian microRNA expression atlas based on small RNA library sequencing. Cell 129: 1401-1414, 2007.

10. Nandy C, Mrázek J, Stoiber H, Grässer FA, Hüttenhofer A and Polacek N: Epstein-barr virus-induced expression of a novel human vault RNA. J Mol Biol 388: 776-784, 2009.

11. Stadler PF, Chen JJ, Hackermüller J, Hoffmann S, Horn F, Khaitovich P, Kretzschmar AK, Mosig A, Prohaska SJ, Qi X, et al: Evolution of vault RNAs. Mol Biol Evol 26: 1975-1991, 2009.

12. Lee HS, Lee K, Jang HJ, Lee GK, Park JL, Kim SY, Kim SB, Johnson BH, Zo JI, Lee JS and Lee YS: Epigenetic silencing of the non-coding RNA nc886 provokes oncogenes during human esophageal tumorigenesis. Oncotarget 5: 3472-3481, 2014.

13. Lee KS, Park JL, Lee K, Richardson LE, Johnson BH, Lee HS, Lee JS, Kim SB, Kwon OH, Song KS, et al: nc886, a non-coding RNA of anti-proliferative role, is suppressed by CpG DNA methylation in human gastric cancer. Oncotarget 5: 3944-3955, 2014.

14. Cao J, Song Y, Bi N, Shen J, Liu W, Fan J, Sun G, Tong T, He J, Shi Y, et al: DNA methylation-mediated repression of miR-886-3p predicts poor outcome of human small cell lung cancer. Cancer Res 73: 3326-3335, 2013.

15. Treppendahl MB, Qiu X, Søgaard A, Yang X, Nandrup-Bus C, Hother $\mathrm{C}$, Andersen $\mathrm{MK}$, Kjeldsen L, Möllgård L, Hellström-Lindberg E, et al: Allelic methylation levels of the noncoding VTRNA2-1 located on chromosome 5q31.1 predict outcome in AML. Blood 119: 206-216, 2012.
16. Kunkeaw N, Jeon SH, Lee K, Johnson BH, Tanasanvimon S, Javle M, Pairojkul C, Chamgramol Y, Wongfieng W, Gong B, et al: Cell death/proliferation roles for nc886, a non-coding RNA, in the protein kinase R pathway in cholangiocarcinoma. Oncogene 32: 3722-3731, 2013

17. Kong L, Hao Q, Wang Y, Zhou P, Zou B and Zhang YX: Regulation of p53 expression and apoptosis by vault RNA2-1-5p in cervical cancer cells. Oncotarget 6: 28371-28388, 2015.

18. Joo MK, Park JJ and Chun HJ: Recent updates of precision therapy for gastric cancer: Towards optimal tailored management. World J Gastroenterol 22: 4638-4650, 2016.

19. Peng HY, Cheng YC, Hsu YM, Wu GH, Kuo CC, Liou JP, Chang JY, Jin SL and Shiah SG: MPT0B098, a microtubule inhibitor, suppresses JAK2/STAT3 signaling pathway through modulation of SOCS3 stability in oral squamous cell carcinoma. PLoS One 11: e0158440, 2016.

20. Lu YM, Chen W, Zhu JS, Chen WX and Chen NW: Eriocalyxin B blocks human SW1116 colon cancer cell proliferation, migration, invasion, cell cycle progression and angiogenesis via the JAK2/STAT3 signaling pathway. Mol Med Rep 13: 2235-2240, 2016.

21. Feng J, Yan PF, Zhao HY, Zhang FC, Zhao WH and Feng M: SIRT6 suppresses glioma cell growth via induction of apoptosis, inhibition of oxidative stress and suppression of JAK2/STAT3 signaling pathway activation. Oncol Rep 35: 1395-1402, 2016.

22. Livak KJ and Schmittgen TD: Analysis of relative gene expression data using real-time quantitative PCR and the 2(-Delta Delta C(T)) method. Methods 25: 402-408, 2001.

23. Yu H, Lee H, Herrmann A, Buettner R and Jove R: Revisiting STAT3 signalling in cancer: New and unexpected biological functions. Nat Rev Cancer 14: 736-746, 2014.

24. Cacalano NA: Regulation of natural killer cell function by STAT3. Front Immunol 7: 128, 2016.

25. Zimmers TA, Fishel ML and Bonetto A: STAT3 in the systemic inflammation of cancer cachexia. Semin Cell Dev Biol 54: 28-41, 2016.

26. Chai EZ, Shanmugam MK, Arfuso F, Dharmarajan A, Wang C, Kumar AP, Samy RP, Lim LH, Wang L, Goh BC, et al: Targeting transcription factor STAT3 for cancer prevention and therapy. Pharmacol Ther 162: 86-97, 2016.

27. Niu G, Wright KL, Ma Y, Wright GM, Huang M, Irby R, Briggs J, Karras J, Cress WD, Pardoll D, et al: Role of Stat3 in regulating p53 expression and function. Mol Cell Biol 25: 7432-7440, 2005.

28. Chung SS and Vadgama JV: Curcumin and epigallocatechin gallate inhibit the cancer stem cell phenotype via down-regulation of STAT3-NFкB signaling. Anticancer Res 35: 39-46, 2015. 